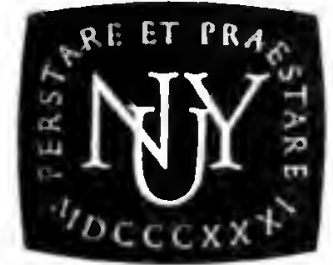

NEW YORK UNIVERSITY

COURANT INSTITUTE OF

MATHEMATICAL SCIENCES

\title{
Automorphic Forms and Poincaré Series for Infinitely Generated Fuchsian Groups
}

\author{
LIPMAN BERS
}

\author{
PREPARED UNDER \\ CONTRACT NO. DA-ARO-(D)-31-124-G156 . \\ WITH THE \\ UNITED STATES ARMY RESEARCH OFFICE
}



New York Undversity

Courant Institute of Mathematical Sciences

AUTOMORPHIC FORMS AND POINCARE SERIES FOR

INFINITELY GENERATED FUCHSIAN GROUPS

Lipman Bers

This report represents results obtained at the Courant Institute of Mathematical Sciences, New York UnIversity, with the United States Army Research Office, Contract No. DA-ARO-(D)-31-124-G156. Reproduction in whole or in part $1 \mathrm{~s}$ permitted for any purpose of the United States Government. 

81. Statement of results.

1. Let $D$ be a simply connected domain in the extended complex plane with at least two boundary points, and $G$ a discrete group of conformal self-mapplngs $z \rightarrow A(z)$ of $D$. If $D$ is the upper halfplane $U$ or the unit disc $\triangle$ the elements $A \in G$ are Mbbius transformations and $G$ is a Fuchsian group (or a Fuchsold group in Poincaré's original terminology since we do not assume $G$ to be finftely generated). While this can be always achieved by a conformal mapping, there are some advantages in considering the seemingly more general case of an arbitrary $D$.

Let

$$
q \geq 2
$$

be a fixed integer. An automorphic form of weight $(-2 q)$ is a holomorphic solution of the functional equation

$$
\phi(A(z)) A^{\prime}(z)^{q}=\phi(z) \text { for } z \in D, \quad A \in G \text {. }
$$

We require in addition that

$$
\phi(z)=O\left(|z|^{-2 q}\right), \quad z \rightarrow \infty \text { if } \infty \in D \text {. }
$$

Let $\lambda_{D}(z)|d z|$ denote the Poincaré metric in $D$. The automorph1c forms with

$$
\|\phi\|_{A_{q}(D, G)}=\iint_{D / G} \lambda_{D}(z)^{2-q}|\phi(z)| d x d y<\infty
$$

form the Banach space $A_{q}(D, O)$ of integrable forms. The automorphic forms with 



$$
\|\phi\|_{B_{q}(D, G)}=\text { ess. sup } \lambda_{D}(z)^{-q}|\phi(z)|
$$

form the Banach space $B_{q}(D, G)$ of bounded forms. For $\phi \in A_{q}(D, G)$, $\psi \in \mathrm{B}_{\mathrm{q}}(D, G)$ the Petersson scalar product is defined by

$$
(\phi, \psi)_{q, G}=\iint_{D / G} \lambda_{D}(z)^{2-2 q} \phi(z) \overline{\psi(z)} d x d y .
$$

In (4) and (6) the integration is performed over an arbitrary fundamental region $\omega$ of $G$ in $D$. This means that $\omega \subset D$ is measurable, mes Int $(\omega)=$ mes $\omega, A\left(z_{1}\right)=z_{2}$ for $z_{1}, z_{2} \in \operatorname{Int}(\omega)$ and $1 d \neq A \in G$, and $D=\int_{A E G} A(\omega)$.

If $G=\{1 d\}$, we write $A_{q}(D), B_{q}(D)$ and $(\phi, \psi)_{q}$ instead of $A_{q}(D, G) B_{q}(D, G)$ and $(\phi, \psi)_{q, G}$ Clearly $A_{q}(D, G) \cap A_{q}(D)=\{0\}$ unless $G$ is finite, while $B_{q}(D, G)$ is always a closed Iinear subspace of $B_{q}(D)$.

2. If $D=U$ and $G$ has a fundamental region of finite nonEuclidean area,

$$
\iint_{D / G} \lambda_{D}(z)^{2} d x d y<\infty,
$$

then $A_{q}(D, G)=B_{q}(D, G)$ is the fintte dimensional space of socalled cusp forms. In the general case we have

Theorem 1. The Petersson product establishes an anti1somorph1sm between $B_{q}(D, G)$ and the dual space to $A_{q}(D, G)$. It is trivial that, for a fixed $\psi \in B_{q}(D, G)$,

$$
\ell(\phi)=(\phi, \psi)_{q, G}
$$


2.7

4
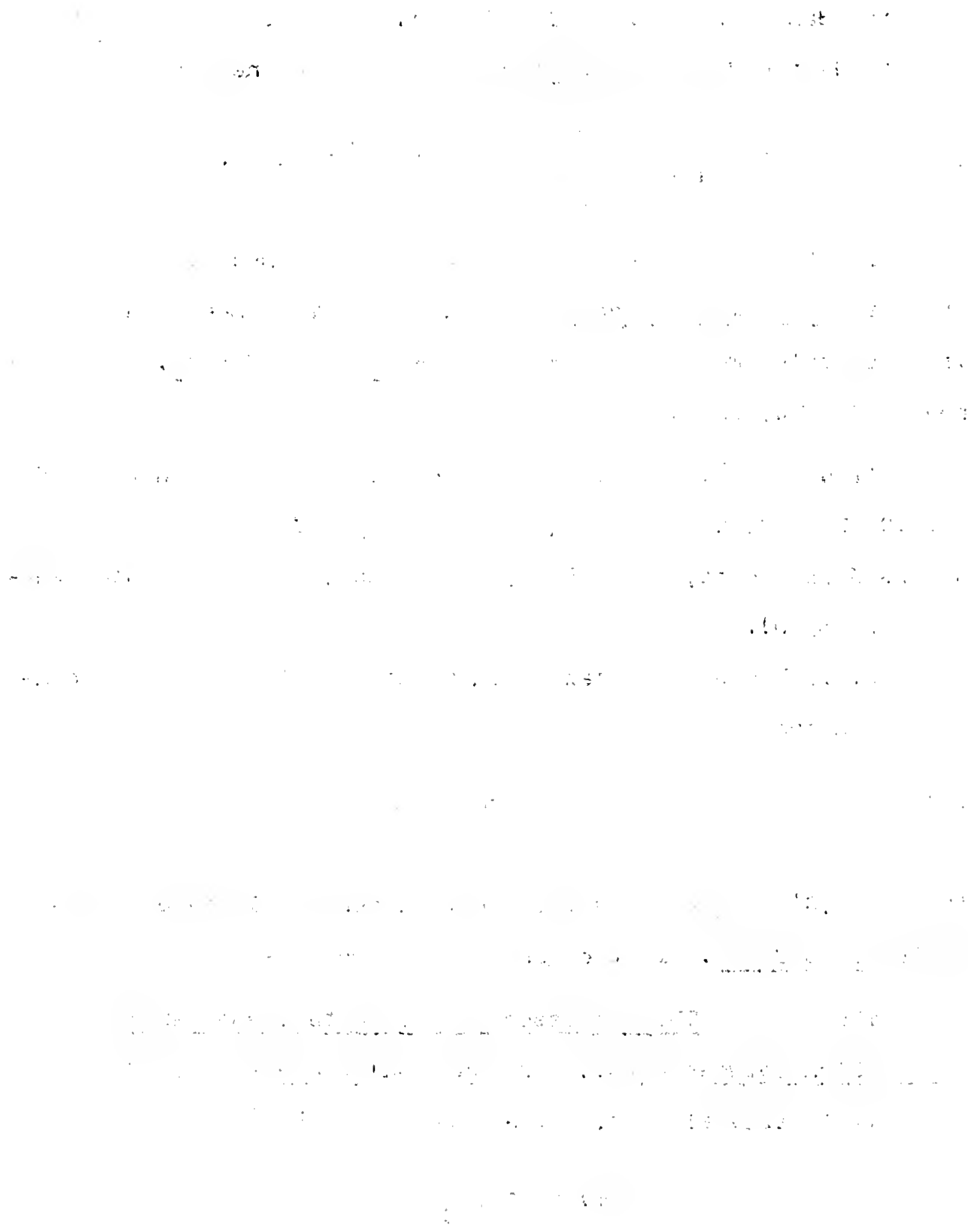
is a continuous inear functional on $A_{q}(D, G)$, of norm $\|\ell\| \leq\|\psi\|_{A_{q}(D, G)}$. To prove Theorem $I$ we will have to show that every $\ell$ can be so represented and that $\psi=0$ whenever $(\phi, \psi)_{a, G}=0$ for all $\phi \in A_{q}(D, G)$.

3. Let $\Phi(z), z \in D$, be a holomorphic function. We say that (⿻) $q, Q \Phi$ exists if

$$
(\because) q, G)(z)=\sum_{A \in G} \Phi(A(z)) A^{\prime}(z)^{q}
$$

where the Poincare series to the right converges absolutely and uniformly on compact subsets of $D$. In this case $(\mathrm{q}, \mathrm{Q} \Phi$ is an automorphic form of weight $(-2 q)$. It is known that if (7) holis, every cusp form is a Poincaré series. In the general case we have

Theorem 2. (A) $q, G$ is a continuous mapping of $A_{q}(D)$ onto $A_{q}(D, G)$.

Thus, for $\Phi \in A_{q}(D), A_{q}, G \Phi$ exists and every $\phi \in A_{q}(D, G)$ is of this form. If $\Phi \in B_{q}(D)$, however, the series in (8) may diverge. It will certainly do so if $G$ is infinite and $\Phi \in B_{q}(D, G)$. Nevertheless we have

Theorem 3. Every $\psi \in B_{q}(D, G)$ is of the form $\psi=A q, G$, $\Phi \in B_{q}(D)$.

Theorems 2 and 3 supercede the results of [2]. For the sake of completeness we shall repeat some arguments from that paper.

4. Assume now that $D=U$ (the upper half-plane). Following Eichler [5] we assign to every automorphic form $\phi$ of weight (-2q) an element of the 1-dimensional cohomology group of $G$ with coefficients in the additive group of polynomials in one variable 

or degree at most 2q-2, the Eichler class of $\phi$ (cr. 20 below). It is known that under hypothesis (7) a cusp form is uniquely determined by its Eichler class.

Theorem 4. If $D=U, G$ is of the first kind, and the Eichler class of $\phi \in \mathrm{B}_{\mathrm{q}}(U, G)$ vanishes, then $\phi=0$.

We recall that $G$ is sald to be of the first or second kind according to whether the whole real axis is or is not contained in the closure $\wedge(G)$ of the set of rixed points of elements of $G$. If $G$ is of the second kind, $\wedge(\cdot G)$ is either a perfect nowhere dense set or contains less than three points. In the latter case $G$ is called elementary.

Theorem 5. Let $D=U$ and let $G$ be a non-elementary group of the second kind. The Eichler class of $\phi \in B_{q}(U, G)$ vanishes if and only if $\phi$ is orthogonal to all forms $M_{q, G} \Phi$, where $\Phi \in A_{q}(U)$ is a rational function with poles in $\Lambda(G)$.

If $G$ is of the second kind, we denote by $A_{2}^{\#}(U, G)$ the set of those $\phi \in \mathrm{A}_{2}(U, G)$ which are continuous and real on the real axis off $\wedge(G)$.

Theorem 6. Let $G$ be as in Theorem 5. The Elchler class of $\phi \in B_{2}(U, Q)$ vanishes if and only if $\phi$ is orthogonal to $A_{2}^{\#}(U, G)$.

In Theorems 5 and 6 orthogonality is meant in the sense of the Petersson product. Theorem 4 and suitably modified forms of Theorems 5 and 6 hold also for $D=\Delta$ (the unit disc).

5. Let $D_{G}$ denote the set $D$ from which the fixed points of elements of $G$ distinct from the identity have been removed. The set $D / G$ has a canonical conformal structure defined by the 

requirement that the projection $D \rightarrow D / G$ be a holomorphic mapping. Thus $D / G$ and $D_{G} / G \subset D / G$ are Riemann surfaces. Let $\pi_{1}$ denote the fundamental group.

Theorem 7. G is finitely generated if and only if $\pi_{I}\left(D_{G} / Q\right)$

1s.

The statement is trivial if $G$ is a fixed point free in $D$, (for then $D=D_{G}$ and since $D$ is simply connected $G$ is 1somorphic to $\left.\pi_{I}(D / G)\right)$. It is "well known" in all cases. But a direct proof has the advantage of enabling one to base the theory of finitely generated Fuchsian groups on uniformization theory to which an easy access via quasi-conformal mappings is now available (cf. [2]). Recently Ahlfors [1] extended Theorem 7 to Klelnian groups. Our proof of Theorem 7 is based on Theorems 4 and 6 . We remark that while the proof of Theorem 4 is almost trivial the reduction of Theorem 6 to Theorem 5 depends on a device employed by Ahlfors.

\section{Preliminaries.}

6. Let $f(z)$ be a conformal mapping of $D$. The Poincare metric has the property that

$$
\lambda_{D}(z)|d z| \text { is a conformal invariant. }
$$

This means that $\lambda_{f(D)}(f(z))\left|f^{\prime}(z)\right|=\lambda_{D}(z)$.

For every $A \in G$ set $\hat{A}=f \circ A \circ f^{-1}$. These $\hat{A}^{\prime} s$ form a discrete group $\hat{G}$ of conformal self-mappings of $f(D)$. For every function $\phi(\zeta), \zeta \in f^{(D)}$, set $\left(f^{*} \phi\right)(z)=\phi(f(z)) f^{\prime}(z)^{q}$. Noting condition (3) we verify that $f^{*}$ is an isometric linear mapping of $A_{q}(f(D), \hat{G})$ 

onto $A_{q}(D, G)$ and of $B_{q}(f(D), \hat{G})$ onto $B_{q}(D, G)$ which preserves the Petersson product:

$$
\left(f^{*} \phi, f^{*} \psi\right)_{q, \hat{G}}=(\phi, \psi)_{q, G} .
$$

One also verifies that

$$
\text { () } \left.\mathrm{q}, \mathrm{G}^{\mathrm{f}^{*} \Phi}=\mathrm{f}^{*}, \widehat{M}\right) \mathrm{q}, \hat{\mathrm{G}} \Phi
$$

where the existence of one side implies that of the other. Hence it suffices to prove Theorems $1-3$ for some fixed domain $D$.

I. We have that

$$
A_{q}(D) \subset B_{q}(D),
$$

this infection being a continuous mapping.

It suffices to prove this for $D=U(c f .6)$ and since

$$
\lambda_{U}(z)=|z-\bar{z}|^{-1}
$$

the assertion follows by a standard estimate:

$$
\begin{aligned}
|\phi(z)| & \leq \frac{4}{\pi y^{2}} \underset{|\zeta-z|<y / 2}{ }|\phi(\zeta)| d \xi d \eta \\
& \leq \frac{4}{\pi y^{2}} \iiint_{|\zeta-z|<y / 2}\left(\frac{2 \eta}{3 y}\right)^{q-2}|\phi(\zeta)| d \xi d \eta \leq \frac{2^{q}}{3^{q-2} \pi y^{2}} \iint_{\eta>0} \eta^{q-2}|\phi(\zeta)| d \xi d \eta
\end{aligned}
$$

so that $\|\phi\|_{B_{q}(U)} \leq 2^{q} 3^{2-q_{\pi}-1}\|\phi\|_{A_{q}(U)}$.

8. The Bergman kernel function $k_{D}(z, \zeta), z \in D, \zeta \in D$ may be defined by the requirements

$$
k_{U}(z, \zeta)=-1 / \pi(z-\bar{\zeta})^{2}
$$





$$
k_{D}(z, \zeta) d z d \bar{\zeta} \text { is a conformal invariant, }
$$

which means that $k_{f(D)}(f(z), f(\zeta)) f^{\prime}(z) \overline{f^{\prime}(\zeta)}=k_{D}(z, \zeta)$ for every conformal mapping $f$ of $D$. The kernel $k_{D}(z, \zeta)$ is a holomorphic function of $z$ and $\bar{\zeta}$ and

$$
k_{D}(\zeta, z)=\overline{k_{D}(z, \zeta)}, \quad \pi k_{D}(z, z)=\lambda_{D}(z)^{2} .
$$

Also,

$$
\iint_{D} \lambda_{D}(\zeta)^{2-q}\left|k_{D}(z, \zeta)\right|^{q} d \xi d \eta=C_{q} \lambda_{D}(z)^{q}
$$

where $C_{q}$ is a constant. In view of (9) and (12) it suffices to verify this for $D=U$ in which case (15) follows from the identity

$$
\iint_{\eta>0} \frac{y^{q} \eta^{q-2} d \xi d \eta}{|x+1 y-\xi+1 \eta|^{2 q}}=\int_{-\infty}^{+\infty} \frac{d \xi}{\left(1+\xi^{2}\right)^{q}} \int_{0}^{+\infty} \frac{\eta^{q-2} d \eta}{(1+\eta)^{2 q-1}}
$$

for $y>0$.

From now on we omit the subscript D. The following reproducing formula holds (as it does also in bounded homogeneous domains in several variables, cf. Selberg [6]):

$$
\phi(z)=c_{q} \iint_{D} \lambda(\zeta)^{2-2 q_{k}(z, \zeta)^{q} \phi(\zeta) d \xi d \eta}
$$

for $\phi \in B_{q}(D)$, where

$$
c_{q}=(2 q-1) \pi^{q-1}
$$

It suffices to verify this for $D=\Delta$. Since 

(17) $k_{\Delta}(z, \zeta)=1 / \pi(1-z \bar{\zeta})^{2}, \quad \lambda_{\Delta}(z)=\left(1-|z|^{2}\right)^{-1}$

and

$$
\text { (2q-1) } \iint_{|\zeta|<1} \frac{\left(1-|\zeta|^{2}\right)^{2 q-2} \zeta^{m} \mathrm{~d} \xi d \eta}{(1-z \bar{\zeta})^{2 q}}=\pi z^{m}, \quad m=0,1, \ldots
$$

the assertion follows.

2. Let $I_{I}(D)$ and $I_{\infty}$ (D) denote the usual complex Banach spaces of (equivalence classes of ) integrable and bounded measurable functions in D. For $\mu \in L_{I}$ (D) set

$$
\left(a_{q} \mu\right)(z)=c_{q} \iint_{D} \lambda(\zeta)^{-q_{k}}(z, \zeta)^{q_{\mu}}(\zeta) d \xi d \eta
$$

and for $v \in L_{\infty}$ (D) set

$$
\left(\beta_{q} \nu\right)(z)=c_{q} \iint_{D} \lambda(\zeta)^{2-q_{k}(z, \zeta)^{q} \nu(\zeta) d \xi d \eta}
$$

By (15) the mappings $\alpha_{q}$ and $\beta_{q}$ are continuous linear mappings of $\mathrm{L}_{1}(D)$ and $\mathrm{L}_{\infty}(D)$ into $\mathrm{A}_{q}(D)$ and $\mathrm{B}_{q}(D)$, respectively. These mappings are onto, since

$$
a_{q}\left(\lambda^{2-q_{\phi}}\right)=\phi \text { for } \phi \in A_{q}(D) \text {, }
$$

$$
\beta_{q}\left(\lambda^{-q} \phi\right)=\phi \text { for } \phi \in B_{q}(D) \text {, }
$$

by $(10)$ and $(16)$. Also

(22) $\quad\left(a_{q^{\mu, \psi}}\right)_{\mathrm{q}}=\iint_{D} \mu(z) \lambda(z)^{-q_{\psi(z)}} \mathrm{dxdy}$ for $\psi \in \mathrm{B}_{\mathrm{q}}(D)$, 



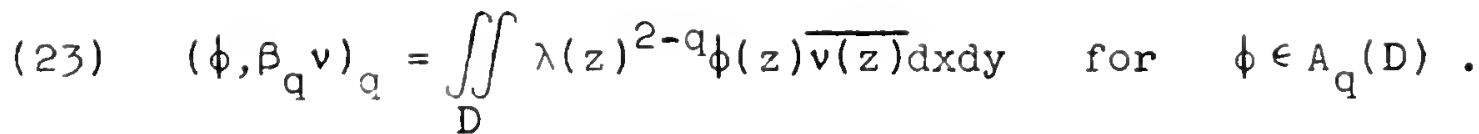

The proof involves merely substitution into the definition (6) for $G=\{1 d\}$, a change of order of integration, and an application of $(16)$.

10. Let $l$ be a continuous linear functional on $A_{q}(D)$. By the theorems of Hahn-Banach and F. Riesz there is a $v \in L_{\infty}$ (D) such that

$$
\dot{\nu}(\phi)=\iint_{D} \lambda_{D}(z)^{2-q_{\phi}} \phi(z) \overline{v(z)} d x d y .
$$

Hence, by (23) we have that $\ell(\phi)=(\phi, \psi)_{q}$ where $\psi=\beta_{q} v$. Next, let $\psi \in B_{q}(D)$ be such that $(\phi, \psi)_{q}=0$ for all $\phi \in A_{q}(D)$. Noting (22) we conclude that

$$
\iint_{D} \lambda(z)-q \overline{\psi(z) \mu}(z) d x d y=0
$$

for all $\mu \in \mathrm{L}_{1}(D)$. Hence $\psi \equiv 0$. Thus we have proved Theorem 1 for the case $G=\{i d\}$.

83. Poincaré series and duality.

11. We prove now that $\left.{ }^{4}\right)_{\mathrm{q}}=\Theta_{\mathrm{q}, G}$ is a continuous mapping of $A_{q}(D)$ into $A_{q}(D, G)$.

Let $\Phi \in A_{q}(D)$ and let $\omega$ denote a fundamental region of $G$ in D. Then 



$$
\begin{aligned}
& \iint_{\omega} \lambda(z)^{2-q}\left|\sum_{A \in G} \Phi(A(z)) A^{\prime}(z)^{q}\right| d x d y \\
& \leq \sum_{A \in G} \iint_{\omega} \lambda(z)^{2-q}\left|\Phi(A(z)) A^{\prime}(z)^{q}\right| d x d y \\
&=\sum_{A \in G} \iint_{\omega} \lambda(A(z))^{2-q} \mid \Phi\left(\left.A(z)|| A^{\prime}(z)\right|^{2} d x d y\right. \\
&=\sum_{A \in G} \iint_{A(\omega)} \lambda(z)^{2-q}|\Phi(z)| d x d y=\|\Phi\|_{A}(D) .
\end{aligned}
$$

This implies the absolute and uniform convergence of the series (8) in every compact subset of a fundamental region and hence on every compact subset of $D$, as well as the inequality

$$
\left\|\Theta_{q} \Phi\right\|_{A}(D, G) \leq\|\Phi\|_{A_{q}}(D)
$$

(Here we used two well known facts: $L_{1}$ convergence of holomorphic functions implies normal convergence. If $\mathrm{D}_{0} \subset \subset \mathrm{D}$ there is an $\omega_{0} \subset \subset \omega$ and a finite sequence $\left\{A_{1}, \ldots, A_{n}\right\} \subset G$ such that $\left.D_{0} \subset A_{1}\left(\omega_{0}\right) \cup \ldots \cup A_{n}\left(\omega_{0}\right).\right)$

12. Let $\ell$ be a continuous linear functional on $A_{q}(D, G)$. Let $\omega$ be a fundamental region. Then, by Hahn-Banach and F. Riesz,

$$
\ell(\phi)=\iint_{\omega} \lambda(z)^{2-q} \phi(z) \nu(z) d x d y
$$

with a bounded measurable $v(z)$. We extend $v$ over the whole of $D$ by the relation

$$
v(A(z))\left(\overline{A^{\prime}(z)} / A^{\prime}(z)\right)^{q / 2}=v(z) \text { for } A \in G
$$

$\left(\right.$ where $\left.\left(\bar{A}^{\prime} / A^{\prime}\right)^{q / 2}=\left|A^{\prime}\right|^{q}\left(A^{\prime}\right)^{-q}\right)$. For $\Phi \in A_{q}(D)$ we have 

(26)

$$
\ell(\AA \Phi)=\iint_{\mathrm{D}} \lambda(z)^{2-q \Phi} \Phi(z) v(z) \mathrm{dxdy}
$$

as follows from the identiy

$$
\begin{aligned}
\iint_{\omega} \lambda(z)^{2-q} \sum \Phi(A(z)) A^{\prime}(z)^{q}{ }_{\nu}(z) d x d y \\
=\sum_{\overline{A \in G}} \iint_{A(\omega)} \lambda(z)^{2-q} \Phi(z) v(z) d x d y .
\end{aligned}
$$

Using this we shall show that

$$
\ell(\hat{q} \Phi)=0 \text { for all } \Phi \in A_{q}(D)
$$

Implies that

$$
\ell(\phi)=0 \text { for all } \phi \in A_{q}(D, G) \text {, }
$$

which means that

$$
\text { (x) } A_{q}(D) \text { is dense in } A_{q}(D, G) \text {. }
$$

During this proof we assume that $D=\Delta$ (the unit disc) and 0 is not a fixed point of any element of G distinct from the identity. This assumption involves no loss of generality.

$$
\text { 13. For } v \text { satisfying (25) for } D=\Delta \text { and such that the }
$$
corresponding functional $l$ vanishes on $\overparen{A} \mathrm{q}^{A}(\Delta)$ set

$$
h(z)=-\frac{1}{\pi} \iiint_{|\zeta|<1} \frac{\left(1-|\zeta|^{2}\right)^{q-2} v(\zeta) d \xi d \eta}{\zeta-z}
$$

and, for some fixed $\theta, 0<\theta<2 \pi$, 

(31) $\tilde{h}(z)=-\frac{\left(1-z e^{-1 \theta}\right)^{q-2}}{\pi} \iint_{|\zeta|<I} \frac{\left(1-|\zeta|^{2}\right)^{q-2} v(\zeta) d \xi d \eta}{\left(1-\zeta e^{-i \theta}\right)^{q-2}(\zeta-z)}$.

For a fixed $z$ such that $|z| \geq 1$ the functions

$$
\Omega(\zeta)=-\frac{1}{\pi} \frac{1}{\zeta-z}, \quad \tilde{\Omega}(\zeta)=-\frac{1}{\pi} \frac{1}{\left(1-\zeta e^{-1 \theta}\right)^{q-2}(\zeta-z)}
$$

belong to $A_{q}(\Delta)$, and by $(26)$

$$
\left.h(z)=\ell(\hat{A} \Omega), \quad \tilde{h}(z)=\left(1-z e^{-1 \theta}\right) q-2 \ell(\Omega) \tilde{\Omega}\right),
$$

so that by (27)

$$
h(z)=\tilde{h}(z)=0 \text { for }|z| \geq 1 \text {. }
$$

From well known properites of logarithmic potentials we conclude that $\mathrm{h}$ and $\tilde{h}$ are continuous everywhere and that, in view of the second equation (32),

$$
\left|\iint_{|\zeta|<1} \frac{\left(1-|\zeta|^{2}\right)^{q-2} v(\zeta) d \xi d \eta}{\left(1-\zeta e^{-i \theta}\right)^{q-2}(\zeta-z)}\right| \leq c(1-|z|) \log \frac{1}{1-|z|}
$$

for $|z|<1$, where $c$ does not depend on $\theta$. Also

$$
\frac{\partial h}{\partial \bar{z}}=\frac{\partial \tilde{h}}{\partial \bar{z}}=\left(1-|z|^{2}\right)^{q-2} v(z) \text { for }|z|<1
$$

(In the sense of weak derlvatives). By (32) and (34) we have that $\mathrm{h} \equiv \tilde{\mathrm{h}}$. Noting (33) and the fact that $\theta$ was aribtrary we conclude that

$$
h(z)=O\left(-(1-|z|)^{q-1} \log (1-|z|)\right), \quad|z| \uparrow 1 .
$$

One computes easily from (34) and (25) that for every fixed $A \in G$ the function 



$$
h(A(z)) A^{\prime}(z)^{l-q}-h(z)
$$

is holomorphic in $|z|<1$. Since it vanishes on $|z|=1$ we have that

$$
h(A(z))=h(z) A^{\prime}(z)^{q-1} \text { for } A \in G \text {. }
$$

Using these properties of $h$ we shall show that $l \equiv 0$.

14. Let $\omega$ be the closure in $\Delta$ of the set

$$
\{z \in \Delta|| A(z)|>| z \mid \text { for } \text { id } \neq A \in G\}
$$

and let $\omega_{r}$ be the intersection of $\omega$ with $|z|<r<1$. Then $\omega$ is a fundamental region. For every $r, 0<r<1$, the boundary $\sigma_{r}$ of $\omega_{r}$ consists of a portion $\gamma_{r}$ of the circle $|z|=r$ and of $2 n=2 n(r)$ circular $\operatorname{arcs} \delta_{1}, \ldots, \delta_{n}, \delta_{1}^{1}, \ldots, \delta_{n}^{\prime}$ such that there exist elements $A_{1}, \ldots, A_{n}$ of $G$ with

$$
A_{j}\left(\sigma_{j}\right)=-\sigma_{j}^{\prime},
$$$$
j=1, \ldots, n
$$

All this is known and easy to check.

Now let $\phi \in A_{q}(\Delta, G)$ be given. By (24) and (34)

$$
\begin{aligned}
\ell(\phi) & =\lim _{r\lceil 1} \iint_{\omega_{r}}\left(1-|z|^{2}\right)^{q-2} v(z) \phi(z) d x d y \\
& =\lim _{r \uparrow 1} \iint_{\omega_{r}} \phi \frac{\partial h}{\partial \bar{z}} d x d y=\frac{1}{2} \underset{r \uparrow 1}{\lim } \int_{\sigma_{r}} \phi h d z .
\end{aligned}
$$

Since by (34) we have that

$$
\phi(A(z)) h(A(z)) A^{\prime}(z)=\phi(z) h(z) \text { for } A \in G \text {, }
$$



so that

$$
\int_{\delta} \phi h d z+\int_{\delta_{j}^{\prime}} \phi h d z=0, \quad j=1, \ldots, n,
$$

and by (35)

$$
-21 l(\phi)=\lim _{\mathrm{r} \uparrow 1} \int_{\gamma_{\mathrm{r}}} \phi \mathrm{hdz}
$$

(38)

$$
|\ell(\phi)| \leq \text { const. } \lim _{r \uparrow 1} \operatorname{Inf}(1-r) \log \frac{1}{1-r} \int_{\gamma_{r}}|\phi||d z| .
$$

Since

$$
\int_{I / 2}^{I}\left(I-r^{2}\right) \int_{\gamma_{r}}|\phi||d z| d r \leq\|\phi\|_{A_{q}}(\Delta, G)
$$

(38) 1mplies that $\ell(\phi)=0$. Q.E.D.

15. For $\Phi \in A_{q}(D), \psi \in B_{q}(D, G)$ we have that

(39)

$$
(\Phi, \psi)_{\mathrm{q}}=\left(\widehat{N}_{\mathrm{q}} \Phi, \psi\right)_{\mathrm{q}, \mathrm{G}} \text {. }
$$

Indeed this means that

$$
\begin{aligned}
\sum_{A \in G} & \iint_{A(\omega)} \lambda(z)^{2-2 q} \Phi(z) \overline{\psi(z)} d x d y \\
& =\iint_{\omega} \lambda(z)^{2-2 q} \overline{\psi(z)} \sum_{A \in G} \Phi(A(z)) A^{\prime}(z)^{a} d x d y
\end{aligned}
$$

which is easily verifled.

16. Proof of Theorem 1. Assume that $\psi \in B_{q}(D, G)$ is such that $(\phi, \psi)_{q, G}=0$ for all $\phi \in A_{q}(D, G)$, then $\left.(A)_{q} \Phi, \psi\right)_{q, G}=0$ for all $\Phi \in A_{q}(D)$ and by (39) also $(\Phi, \psi)_{q}=0$. Hence $\psi=0$ by the result In 10 .

Now let $l(\phi)$ be a given linear functional on $A_{q}(D, G)$. Then (cf. 12) there is a $v \in L_{\infty}$ (D) satisfying (25) such that (24) holds. 

Set $\psi=\beta_{\mathrm{q}} \bar{v}$. Then (cf. 9) $\quad \psi \in \mathrm{B}_{\mathrm{q}}(\mathrm{D})$ and by (26) and (23)

(40)

$$
\ell\left(\hat{\sigma}_{\mathrm{q}} \Phi\right)=(\Phi, \psi)_{\mathrm{q}} \text { for } \Phi \in \mathrm{A}_{\mathrm{q}} \text {. }
$$

Now, for $A \in G$ and $B=A^{-1}$

$$
\psi(A(z)) A^{\prime}(z)^{q}=c_{q} \iint_{D} A^{\prime}(z)^{q} \lambda(\zeta)^{-q_{k}(z, \zeta)^{q}} \overline{v(\zeta)} d \xi d \eta .
$$

Setting $\zeta=B \hat{\zeta}$ and notine $(9),(13)$ we obtain

$$
\begin{aligned}
& \psi(A(z)) A^{\prime}(z)^{q}=c_{q} \iint_{D} A^{\prime}(z)^{q} \lambda(\zeta)^{2-q_{k}(A(z), \zeta)^{q} \overline{v(\zeta)}} d \xi d \eta \\
& =c_{q} \iint_{D} \lambda(A \circ B(\zeta))^{2-q_{A^{\prime}}(z)^{q_{k}}} d(z(z), A \circ B(\zeta))^{q} \overline{v(A \circ B(\zeta))} d \xi d \eta \\
& =c_{q} \iint_{D} \lambda(B(\zeta))^{2-q_{k}(z, B(\zeta))^{q} \overline{v(B(\zeta))}\left|B^{\prime}(\zeta)\right|^{2} d \xi d \eta=\psi(z) .}
\end{aligned}
$$

Thus $\psi \in \mathrm{B}_{\mathrm{q}}(D, G)$ and, by $(39)$ and $(40)$,

$$
\ell(\phi)=(\phi, \psi)_{\mathrm{q}, \mathrm{G}}
$$

whenever $\phi \in A q^{A} q(D)$. In view of (29) the same holds for all $\phi \in A_{q}(D, G)$.

17. Proof of Theorem 2. In view of 11 we must show only that (A) $\mathrm{q}_{\mathrm{q}}(D)=\mathrm{A}_{\mathrm{q}}(D, G)$. Let $X$ be the characteristic function of a fundamental region $\omega$. Then $x \lambda^{2-q_{\phi} \in I_{\infty}}(D)$ and we may form $\hat{\phi}=\Theta_{q} \alpha_{q}\left(\chi \lambda^{2-q_{\phi}}\right)$ which belongs to $A_{q}(D, G)$. Let $\psi$ be any element in $B_{q}(D, G)$. By (39) 

$(\hat{\phi}, \psi)_{q, G}=\left(a_{q}\left(\not \lambda^{\left.\left.2-q_{\phi}\right), \psi\right)_{q}}\right.\right.$

$$
\begin{aligned}
& =c_{q} \iint_{D} \lambda(z)^{2-2 q} \overline{\psi(z)} \iint_{\omega} \lambda(\zeta)^{2-2 q} \phi(\zeta) k(z, \zeta)^{q} d \xi d \eta d x d y \\
& =c_{q} \iint_{\omega} \lambda(\zeta)^{2-2 q} \phi(\zeta) \iint_{D} \lambda(z)^{2-2 q} \overline{k(\zeta, z)} \overline{\psi(z)} d x d y d \xi d \eta \\
& =(\phi, \psi)_{q, G} .
\end{aligned}
$$

Hence

(41)

$$
\phi=q_{q} q_{q}\left(x \lambda^{2-a} \phi\right),
$$

by Theorem 1 .

18. Proof of Theorem 3. Let $\chi$ be as in the previous proof. We shall show that if $\phi \in \mathrm{B}_{\mathrm{q}}(D, G)$, then

$$
\phi=\Omega q_{q}^{\beta}\left(x_{\lambda}-q_{\phi}\right)
$$

(note that $\left.\chi_{\lambda}-q_{\phi \in L_{\infty}}(D)\right)$. By (16)

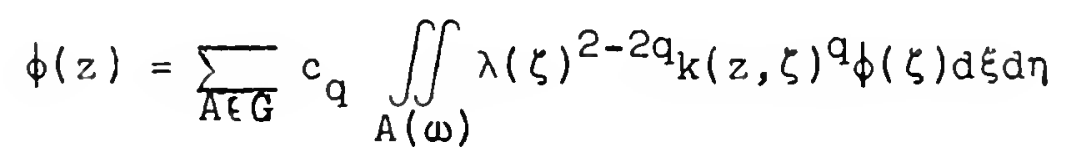

this series being absolutely and normally convergent. Setting $B=A^{-1}$ and using (2), (9) and (12) we obtain

$$
\begin{aligned}
\phi(z) & =\sum_{A \in G} c_{q} \iint_{A(\omega)} \lambda(B(\zeta))^{2-2 q_{k}(B(z), B(\zeta))^{q} \phi(B(\zeta)) B^{\prime}(z)^{q}\left|B^{\prime}(\zeta)\right|^{2} d \xi d \eta} \\
& =\sum_{A \in G} c_{q} B^{\prime}(z)^{q} \iint_{\omega} \lambda(\zeta)^{2-2 q_{k}(B(z), \zeta)^{q} \phi(\zeta) d \xi d \eta}
\end{aligned}
$$

which is precisely (42). 

84. Periods of automorphic forms

19. Let $D=U$ so that $G$ is a group of Mbbius transformations $z \rightarrow A(z)=(a z+b) /(c z+d)$. Let $\prod_{2 q-2}$ denote the additive groups of polynomials $P(z)=\sum_{j=0}^{2 q-2} \alpha_{j} z^{j}$. The group $G$ operates from the right on $\prod_{2 q-2}$ by the rule

$$
(P A)(z)=P(A(z)) A^{\prime}(z)^{1-q} \text {. }
$$

A mapping $A \rightarrow P_{A}$ of $G$ into $\Pi_{2 q-2}$ is called a cocycle of

$$
P_{A B}=P_{A} B+P_{B}
$$

a coboundary if there exists an element $Q \in T_{2 q-2}$ such that

$$
P_{A}=Q A-Q
$$

The coboundaries form a subgroup of the additive group of cocycles. The factor group (cocycles/coboundaries) is denoted by $H^{1}\left(G, \prod_{2 q-2}\right)$.

20. Let $\phi$ be an automorphic form of weight $(-2 q)$ and $F$ a holomorphic function such that

$$
\frac{d^{2 q-1} F(z)}{d z^{2 q-1}}=\phi(z)
$$

One verifies easily that for every $A \in G$ the $(2 q-1)$-st derivative of

$$
F(A(z)) A^{\prime}(z)^{1-q}-F(z)
$$

vanishes, so that this function belongs to $\Pi_{2 q-2}$. We call it the Elchler period of $F$ on $A$. The mapping 



$$
A \rightarrow F(A(z)) A^{\prime}(z)^{1-q}-F(z)
$$

is clearly a cocycle. Since $F$ is determined by $\$$ modulo a polynomlal of degree at most $2 q-2$, the cohomology class of (48) depends only on $\phi$ and depends on $\phi$ linearly. We call it the Eichler class of $\phi$.

The existence of an F satisfying (46) and the condition

$$
F(A(z)) A^{\prime}(z)^{l-q}=F(z) \text { for all } A \in G
$$

Is necessary and sufficlent for the vanishing of the Eichler class of $\phi$.

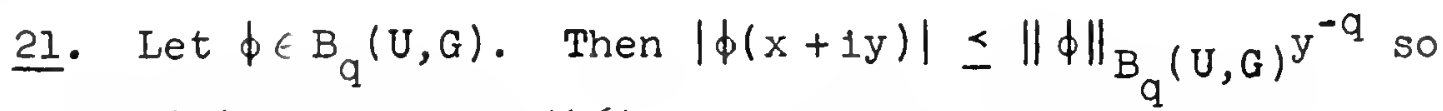
that every $F(z)$ satisfying (46) is continuous on the real axis. Assume that (49) holds and let $x \in \mathbb{R}$ be a fixed point of a hyperbolic parabola element $A$ of $G$. Then $A(x)=x, A^{\prime}(x) \neq I$ and, by (49), $F(x)=0$. Hence also

$$
F(x)=0 \text { for } \quad x \in \wedge(G)
$$

where $\Lambda(G)$ is the closure of the set of fixed points. Conversely, if (46) and (50) hold, then for every fixed $A \in G$ the polynomial (47) vanishes on $\Lambda(G)$ since $A(\Lambda(G))=\Lambda(G)$ for every $A \in G$. If $G$ is not elementary, $\wedge(G)$ is infinite and we conclude that (49) holds.

22. Proof of Theorem 4. If $G$ is of the first kind and the Eichler class of $\phi \in B_{q}(U, G)$ is zero, then $\phi(z)=F^{(2 q-1)}(z)$ with $F=0$ on $\mathbb{R}$. Hence $F \equiv 0, \phi \equiv 0$.

23. Let $\wedge$ be a perfect set on the real axis (in the next paragraph we shall take $\Lambda=\wedge(G)$ for a non-elementary group $G$ of 

the second $k$ ind). Let $a_{1}, \ldots, a_{q}$ be distinct points of $\Lambda$ and set

$$
p(z)=\left(z-a_{1}\right)\left(z-a_{2}\right) \cdots\left(z-a_{q}\right)
$$

Then every rational function with simple poles in $\Lambda$ which belongs to $A_{q}(U)$ is of the form

$$
\sum_{j=I}^{n} \frac{\alpha_{j}}{\left(z-x_{j}\right) p(z)}
$$

where $x_{1}, \ldots, x_{n}$ are distinct points of $\Lambda$ and $x_{j} \neq a_{k}$ and the $a_{j}$ are arbitrary complex constants. Indeed, a rational function with no singularities except perhaps for simple poles at $a_{1}, \ldots, a_{q}$, $x_{1}, \ldots, x_{n}$ belongs to $A_{q}(U)$ if and only if it is of the form

$$
\sum_{j=1}^{\mathrm{q}} \frac{\beta_{j}}{2-a_{j}}+\sum_{j=1}^{n} \frac{\gamma_{j}}{z-x_{j}}
$$

with

$$
\sum_{j=1}^{q} \beta_{j} a_{j}^{s}+\sum_{j=1}^{n} \gamma_{j} x_{j}^{s}=0, \quad s=0,1, \ldots, q-1 .
$$

The space of such functions has therefore dimension $n$. On the other hand (52) always belongs to $A_{q}(U)$.

If $\Phi(z) \in A_{q}(U)$ is a rational function with poles in $\wedge$ it is a limit of functions of the form (52). Indeed, if $\xi_{1}, \ldots, \xi_{m}$ are the poles of $\Phi$ and $v_{1}, \ldots, v_{m}$ their multiplicities we have $0<v_{j} \leq q-1$ and

$$
\Phi(z)=r(z) \prod_{j=1}^{m}\left(z-\xi_{j}\right)^{-v} j
$$

where $r(z)$ is a polynomial of degree at most $v_{1}+\ldots+v_{m}+q-1$ with $r\left(\xi_{j}\right) \neq 0$. Let $\varepsilon>0$ be given. Since $\wedge$ is perfect there exist 
. 
distinct points $\xi_{j k}, j=1, \ldots, m, k=1, \ldots, v_{j}$ in $\wedge$ with $\left|\xi_{j k}-\xi_{j}\right|<\varepsilon$. The function

$$
\tilde{\Phi}(z)=r(z) \prod_{j=1}^{m} \prod_{k=1}^{v}\left(z-\xi_{j k}\right)^{-1}
$$

is of the form (52) and one verifles that $\|\Phi-\Phi\|_{A_{q}}(U)$ will be arbitrarily small for $\varepsilon$ sufficiently amall.

24. Proof of theorem 5. Let $\Lambda=\Lambda(G)$ and let $a_{1}, \ldots, a_{q}$ and $p(z)$ be as in 23. Let $\phi \in B_{q}(U, G)$. Noting (II), (12) we write (16) In the form

Set

$$
\phi(z)=\frac{(-1)^{\mathrm{q}}(2 \mathrm{q}-1)}{\pi} \iint_{\eta \rightarrow 0} \frac{|\zeta-\bar{\zeta}|^{2 q-2} \phi(\zeta) \mathrm{d} \xi \mathrm{d} \eta}{(\bar{\zeta}-z)^{2 \mathrm{q}}} .
$$

$$
G(z)=\iint_{\eta=0} \frac{|\zeta-\bar{\zeta}|^{2 q}-2 \phi(\zeta) \mathrm{d} \xi \mathrm{d} \eta}{(\bar{\zeta}-z) p(\bar{\zeta})}
$$

This function is holomorphic in $U$ and continuous everywhere except perhaps at the points $a_{j}$. Next, set

$$
F(z)=\frac{(-1)^{q} p(z) G(z)}{\pi(2 q-2) !}
$$

Then $F\left(a_{j}\right)=0, j=1, \ldots, q$ and since

$$
\frac{p(z)}{p(\bar{\zeta})(\bar{\zeta}-z)}-\frac{1}{\bar{\zeta}-z}
$$

is a polynomial of degree $q-1$ in $z$, we have that

$$
F^{(2 q-1)}(z)=\frac{(-1)^{q}(2 q-1)}{\pi} \iint_{\eta>0} \frac{|\zeta-\zeta|^{2 q-2} \phi(\zeta) d \xi d \eta}{(\zeta-z)^{2 q}}
$$



In $U$, so that (46) holds. By 21 the Elchler class of $\phi$ vanishes if and only if $F(x)=0$ for $x \in \Lambda(G), x \neq a_{j}$. This condition is equivalent to

$$
G(x)=0 \text { on } \wedge(G)-\left\{a_{1}, \ldots, a_{q}\right\}
$$

But for a real $x \neq a_{j}$

$$
\overline{\pi \overline{G(x)}}=(-1)^{\mathrm{q}}(2 \mathrm{q}-1)(\Phi, \phi)_{\mathrm{q}}
$$

where

$$
\Phi(x)=\frac{1}{(z-x) p(z)} \in A_{q}(U)
$$

The conclusion of Theorem 5 now follows from 23 .

25. Let $G$ be again a Fuchsian non-elementary group of the second k1nd and let $\Omega$ denote the complement of $\Lambda(G)$ in the extended complex plane. Then there exists a Fuchsian group $\mathrm{H}_{\mathrm{O}}$ without elliptic elements and a holomorphic mapping $\zeta \rightarrow \mathrm{g}(\zeta)$ of $U$ onto $\Omega$ such that if $\zeta_{I}, \zeta_{2} \in U$, then $g\left(\zeta_{I}\right)=g\left(\zeta_{2}\right)$ if and only if there is a $\mathrm{C} \in \mathrm{H}_{0}$ with $\mathrm{C}\left(\zeta_{1}\right)=\zeta_{2}$. Also, there is a Fuchsian group $H$ such that if $\zeta_{1}, \zeta_{2} \in U$, then $A\left(g\left(\zeta_{1}\right)\right)=g\left(\zeta_{2}\right)$ for some $A \in G$ if and only if there is a $B \in H$ with $B\left(\zeta_{1}\right)=\zeta_{2}$. The mapping $\tau$ of $H$ onto $G$ which sends $B \in H$ into $A \in G$ with $g \circ B=A \circ g$ is a holomorphism; 1ts kernel is precisely $\mathrm{H}_{0}$.

Let $\phi \in A_{2}^{\prime}(U, G)$. This means that $\phi \in A_{2}(U, G)$ and $\phi(z)$ is holomorphic in $\Omega$ and satisfies the relation

$$
\phi(\bar{z})=\overline{\phi(z)} \text {. }
$$

Let $\omega$ be a fundamental region for $G$ in $\Omega$ chosen so that $\omega \cap U$ is simply connected and $\omega$ is invariant under the mapping $z \rightarrow \bar{z}$. 

Then there is a fundamental region $\hat{\omega}$ for $H$ in $U$ such that $g(\hat{\omega})=\omega$. Let $K \in \mathrm{H}$ contain exactly one representative of each coset of $\mathrm{H}$ modulo $\mathrm{H}_{\mathrm{O}}$. Then

$$
\hat{\omega}_{0}=\bigcup_{B \in K} B(\hat{\omega})
$$

is a fundamental region for $H_{0}$ in $U$ and $g\left(\hat{\omega}_{0}\right)=\Omega$.

$$
\begin{aligned}
& \text { Set } \hat{\phi}(\zeta)=\phi(g(\zeta)) g^{\prime}(\zeta)^{2} \text {. Then } \\
& \iint_{\omega}|\hat{\phi}(\zeta)| d \xi d \eta=\iint_{\omega}|\phi(z)| d x d y=2\|\phi\|_{A_{2}(U, G)}
\end{aligned}
$$

by (53), and for $B \in H$ we have that

$$
\begin{aligned}
\hat{\phi}(B(\zeta)) B^{\prime}(\zeta)^{2} & =\phi\left(g(B(\zeta)) g^{\prime}(B(\zeta))^{2} B^{\prime}(\zeta)^{2}\right. \\
& =\phi\left(A(g(\zeta)) A^{\prime}(g(\zeta))^{2} g^{\prime}(\zeta)^{2}=\phi(g(\zeta)) g^{\prime}(\zeta)^{2}=\hat{\phi}(\zeta)\right.
\end{aligned}
$$

where $A$ is the image of $B$ under the homomorphism $\tau$ described above. Hence $\hat{\phi} \in A_{2}(U, H)$ and by Theorem 2 we have that $\hat{\phi}=(B){ }_{2, H} \Phi$, $\hat{\Phi} \in A_{2}(U)$, or

$$
\begin{aligned}
\hat{\phi}(\zeta) & =\sum \hat{\Phi}(B(\zeta)) B^{\prime}(\zeta)^{2} \\
& =\sum_{B \in K} \sum_{C=H_{O}} \hat{\Phi}\left(C(B(\zeta)) C^{\prime}(B(\zeta))^{2} B^{\prime}(\zeta)^{2} .\right.
\end{aligned}
$$

Set

$$
\hat{\Phi}_{0}(\zeta)=\sum_{C \in H_{0}} \hat{\Phi}(c(\zeta)) c^{\prime}(\zeta)^{2} \text {. }
$$

Then $\hat{\Phi}_{0}=\Theta_{2, H_{0}} \hat{\Phi} \in A_{2}\left(U, H_{0}\right)$. Hence there exists a holomorphic function $\Phi_{0}(z), z \in \Omega$ such that $\hat{\Phi}_{0}(\zeta)=\Phi_{0}(g(\zeta)) g^{\prime}(\zeta)^{2}$; we have that 



$$
\iint_{\Omega}\left|\Phi_{0}(z)\right| d x d y<\infty
$$

since this integral equals $\left\|\hat{\Phi}_{0}\right\|_{A_{2}}\left(U, H_{0}\right)$.

Now (54) may be written as

$$
\begin{aligned}
\phi(g(\zeta)) g^{\prime}(\zeta)^{2} & =\sum_{B \in K} \Phi_{0}(B(\zeta)) B^{\prime}(\zeta)^{2} \\
& =\sum_{B \in K} \Phi_{0}(g(B(\zeta))) g^{\prime}(B(\zeta))^{2} B^{\prime}(\zeta)^{2} \\
& =\sum_{A \in G} \Phi_{0}(A(g(\zeta))) A^{\prime}(g(\zeta))^{2} g^{\prime}(\zeta)^{2}
\end{aligned}
$$

Thus every $\phi \in A_{2}^{\#}(U, G)$ admits the representation

$$
\phi=\omega_{2, G} \Phi_{0}
$$

where $\Phi_{0}$ is holomorphic in $\Omega$ and absolutely integrable over this domain.

26. Proof of Theorem 6. Assume that $\psi \in B_{2}(U, G)$ is orthogonal to $A_{2}^{\#}(U, G)$. Let $r(z)$ be a rational function with poles in $\wedge(G)$ belonging to $A_{2}(U)$ and $\phi=\overbrace{2, G}$. Since

$$
\iint_{\Omega}|r(z)| d x d y<\infty
$$

( $\Omega$ having the same meaning as in 25 ) the argument in 11 can be repeated to show that the Poincaré series

$$
\sum_{A \in G} r(A(z)) A^{\prime}(z)^{2}
$$

converges absolutely and normally in $\Omega$. This implies that $\phi(z)=\phi_{1}(z)+1 \phi_{2}(z)$, with $\phi_{1}, \phi_{2} \in A_{2}^{\text {位 }}(U, G)$. Hence $(\phi, \psi)_{2, G}=0$. By Theorem 5 the Elchler class of $\psi$ is zero. 

Assume next that the E1chier class of $\psi \in B_{2}(U, G)$ vanishes and let $\phi \in A_{2}^{\not /}(U, G)$. Then $\phi$ admits the representation (55). By the approximation theorem proved in [2] there exists a sequence of rational functions $\left\{r_{j}(z)\right\}$ with poles in $\Lambda(G)$ such that

$$
\iint_{\Omega}\left|r_{j}(z)-\Phi_{0}(z)\right| d x d y \rightarrow 0 \text {. }
$$

By Theorem 5 we have that $\left(\Theta_{2, G} r_{j}, \psi\right)_{2, G}=0$. Since $(56)$ implies that $\left\|r_{j}-\Phi_{0}\right\|_{A_{2}}(U) \rightarrow 0$, we have that $\left(A_{2}, G r_{j} \rightarrow \phi\right.$ in $A_{2}(U, G)$, by Theorem 2. Therefore $(\phi, \psi)_{2, G}=0$.

85. Finitely generated Fuchsian groups

27. A Rlemann surface $S$ will be called of finite type, more precisely of type $(\mathrm{g}, \mathrm{n}, \mathrm{m})$, if it is conformally equivalent to $S_{0}-\sigma$ where $S_{0}$ is a closed (compact) surface of genus $g$ and $\sigma a$ closed set w1th $n+m \geq 0$ components of which $n \geq 0$ are points and $m \geq 0$ simply connected non-degenerate continua. The numbers $g, n$, $\mathrm{m}$ depend only on $\mathrm{S}$; we say that $\mathrm{S}$ has $\mathrm{n}$ punctures and $\mathrm{m}$ boundary curves.

$$
\text { If } m=0 \text { then } S_{0} \text { (the natural compactification of } S \text { ) is }
$$
determined by $S$ except for conformal equivalence. If $m>0$ there exists a Riemann surface $S_{1}$ of type $(2 g+m-1,2 n)$ (the double of $S$ ) which is determined by $S$ except for conformal equivalence, $m$ disjoint simple closed analytic curves $\gamma_{1}, \ldots, \gamma_{m}$ on $S_{I}$ and an anticonformal involution $\rho$ of $S_{1}$ which leaves a point $p \in S_{1}$ fixed if and only if $\mathrm{p} \in \gamma=\gamma_{1} \cup \ldots \cup \gamma_{\mathrm{m}}$, such that $\mathrm{s}_{1}-\gamma$ consists of two components one of which is conformally equivalent to $S$. 

The fundamental group $\pi_{1}(S)$ is finitely generated if and only if $S$ is of finlte type. Th1s is a known result in surface topology.

28. Let $D_{G} / G$ be of finite type. Then $G$ is finitely generated.

Th1s 1 s well known and can be proved by disecting $D_{G} / G$ by finitely many smooth curves into a simply connected region such that a component of its inverse image under the projection $D_{G} \rightarrow D_{G} / G$ is a fundamental domain whose boundary consists of finitely many "sides".

29. Let $S$ be a Riemann surface. An Abelian differential (of the first kind) on $S$ is a rule associating with every local $p \rightarrow t(p)$ defined on a domain $G \subset S$ a holomorphic function $\phi(t)$ such that $\phi(t) d t$ is invariant under parameter changes. In this case $|\phi(t)|^{2}$ is a density. If we demand instead the invariance of $\phi(t) d t^{2}$ we obtain a quadratic (holomorphic) differential; now $|\phi(t)|$ is a density. The Abelian differentials $\alpha$ with

$$
\iint_{S}|\alpha|^{2}<\infty
$$

form a Hilbert space $A_{1}(S)$ of square integrable differentials. The quadratic differentials $\beta$ with

$$
\iint_{S}|B|<\infty
$$

form the Banach space $A_{2}(S)$ of integrable differentials. We have that

$$
\operatorname{dim} A_{1}(S) \leq \operatorname{dim} A_{2}(S)
$$




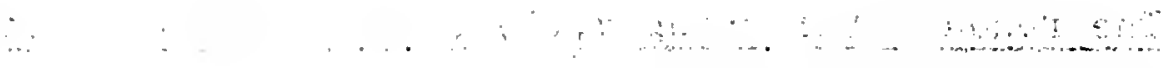

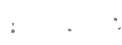

$\therefore \quad \because \quad \because \quad \because \cdots+\cdots$

$\because \quad \therefore \quad$ i

$\because \quad+\quad \cdots+\cdots$

- . $.1, \cdots \cdots$

$\therefore \cdots$

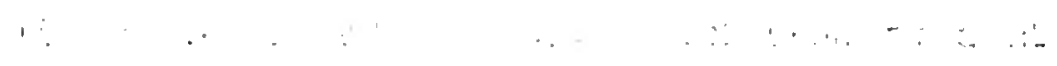

$, \cdots, \cdots, \cdots, \cdots, \cdots, \cdots$

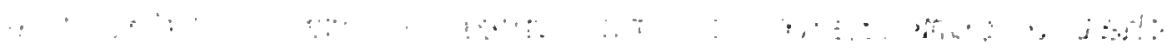

$\because+\therefore, \cdots+\cdots$

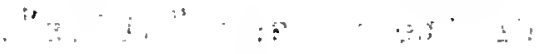

$\therefore \ldots \quad \cdot \cdots$

$\therefore \quad \therefore \quad \cdots \quad \cdot \quad \cdots \quad:$

$\therefore \quad \therefore \quad \therefore \quad \therefore \quad \therefore \quad \therefore \quad \therefore \quad \therefore$

$\therefore \quad(1$

$\therefore$

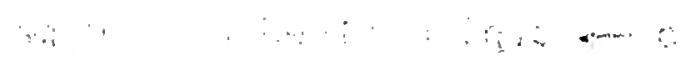

$\therefore \quad \because \quad$ ฯ

$\therefore \quad \therefore \quad \cdots, \quad$ a

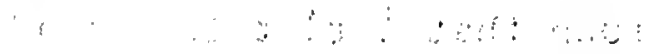

$\therefore \quad: \quad$;

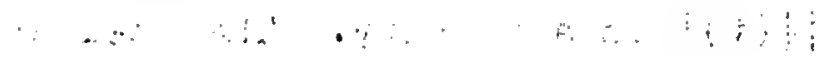

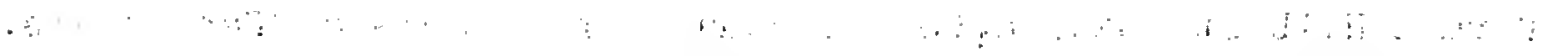

$\therefore \quad \therefore \quad \therefore \quad \therefore \quad \therefore$

$\checkmark \quad \vdots \quad \vdots$

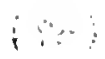

$\therefore \quad: \quad: \quad: \quad ; \quad$ i 
because if $\alpha_{1}, \alpha_{2} \in A_{1}(S)$, then $\alpha_{1} \alpha_{2} \in A_{2}(S)$. If the genus of $S$ is infinite, then $\operatorname{dim} A_{1}(S)=\infty$ (cf. Nevanlinna [4]) and hence $\operatorname{dim} A_{2}(S)=\infty$. If the genus of $S$ is $g<\infty$, then $S=S_{0}-\sigma$ where $\sigma$ is a closed set on the closed surface $S_{0}$ of genus $g$. If $S$ contains $N$ distinct points, then $\operatorname{dim~} A_{2}(S) \geq N$ since it is known (say from the Riemann-Roch theorem) that to every $\mathrm{p} \in \mathrm{S}_{\mathrm{O}}$ there is a meromorphic quadratic differential $\beta_{p}$ on $S_{O}$ whose only singularity is a simple pole at $p$. We conclude that

(58) $\operatorname{dim} A_{2}(S)=\infty$ unless $S$ is of finite type $(g, n, 0)$.

30. The space $A_{2}(D, G)$ can be defined even when $G$ is a discrete group of conformal self-mappings of a non-simply connected domain (since $\lambda$ does not enter in the definition of this space). Let $D_{G}$ denote $D$ with the fixed points of elements of $G$ (distinct from the identity) removed. Then there is a canonical isomorphism

$$
A_{2}(D, G) \cong A_{2}\left(D_{G} / G\right)
$$

Indeed, $A_{2}(D, G)$ may be identified with the space $X$ of meromorphic quadratic differentials $\beta$ on the Rlemann surface $D / G$ for which (57) holds and which have no singularities except perhaps simple poles on the set $\sigma$ consisting of the images of fixed points of $G$ under the profection $D \rightarrow D / G$. Since $\sigma$ is discrete and $D / G-\sigma=D_{G} / G$, X may be identified with $A_{2}\left(D_{G} / G\right)$.

31. Let $G$ be a Fuchsian group. The elements of $B_{q}(U, G)$ with vanishing Eichler class form a closed Iinear subspace $B_{q}^{O}(U, G)$. If $G$ is finitely generated, $\operatorname{dim} B_{q}(U, G) / B_{q}^{O}(U, G)<\infty$. 

Indeed, assign to every $\phi \in B_{q}(U, G)$ a holomorphic function $F(z), z \in U$ such that $F^{(2 q-1)}(z)=\phi(z)$ and $F^{(\nu)}(z)=0$, $v=0,1, \ldots, 2 \mathrm{q}-2$. Then $\phi \in \mathrm{B}_{\mathrm{q}}^{\circ}(U, G)$ whenever the Eichler periods of $F$ vanish on a set of generators of $G$. This amounts to finitely many linear conditions.

32. Proof of Theorem 7. We may assume that $D=U$. We may assume that $G$ is non-elementary, the theorem being trivial for elementary groups. In view of $27, \underline{28}$ it suffices to assume that $G$ is finitely generated and to prove that $U_{G} / G$ is of finite type.

Let $G$ be of the first kind. Then $B_{2}^{O}(U, G)=\{0\}$ by Theorem 4, hence $\operatorname{dim} B_{2}(U, G)<\infty$ by 31 , hence $\operatorname{dim} A_{2}(U, G)<\infty$ by Theorem 1 , hence $\operatorname{dim} A_{2}\left(U_{G} / G\right)<\infty$ by $(59)$, hence $U_{G} / G$ is of the finite type $(g, n, 0)$ by $(58)$.

Assume next that $G$ is of the second kind. Let $A_{2}^{b}(U, G)$ denote the subspace of $\mathrm{A}_{2}(U, G)$ consisting of elements of the form $\phi_{1}+1 \phi_{2}$ with $\phi_{1}, \phi_{2} A_{2}^{i \neq}(U, G)$. By Theorems 1 and 6 the dual space to $A_{2}^{b}(U, G)$ is anti-isomorphic to $B_{2}(U, G) / B_{2}^{O}(U, G)$. Thus $\operatorname{dim} A_{2}^{b}(U, G)<\infty$. Let $\Omega$ have the same meaning as $1 n \underline{25}$. One sees at once that $A_{2}^{b}(U, G)$ may be identified with $A_{2}(\Omega, G)$. Hence $\operatorname{dim} A_{2}\left(\Omega_{G} / G\right)<\infty$ by $(59)$, and in view of $(58)$ the Riemann surface $S_{1}=\Omega_{G} / G$ is of finite type $(g, n, 0)$. The mapping $z \rightarrow \bar{z}$ induces an anti-conformal involution $p$ on $S_{1}$. The set $\gamma$ of fixed points of $\rho$ is the image of the intersection of $\Omega_{G}$ with the extended real axis under the canonical mapping $\Omega_{G} \rightarrow S_{1}$ and one of the two components of $S_{1}-\gamma$ is $U_{G} / G$. Hence $U_{G} / G$ is of finite type $(g, n, m)$ with $n>0$. 



\section{$\underline{\text { References }}$}

1. L. V. Ahlfors, Finitely generated Kleinian groups. To appear.

2. L. Bers, Completeness theorems for Poincare series in one variable. Proc. Int. Symp. on Linear Spaces. Jerusalem Acad. Press - Pergamon Press, 1961, pp. 88-100.

3. L. Bers, An approximation theorem. To appear.

4. R. Nevanlinna, Uniformisierung. Springer, 1953.

5. M. Eichler, Eine Verallgemeinerung der Abelschen Integrale. Math. Z., , pp.

6. A. Selberg, Automorphic functions and integral operators. Seminars on Anal. Functions, Inst. Adv. Study, 1957, vol. II, pp. $152-161$. 


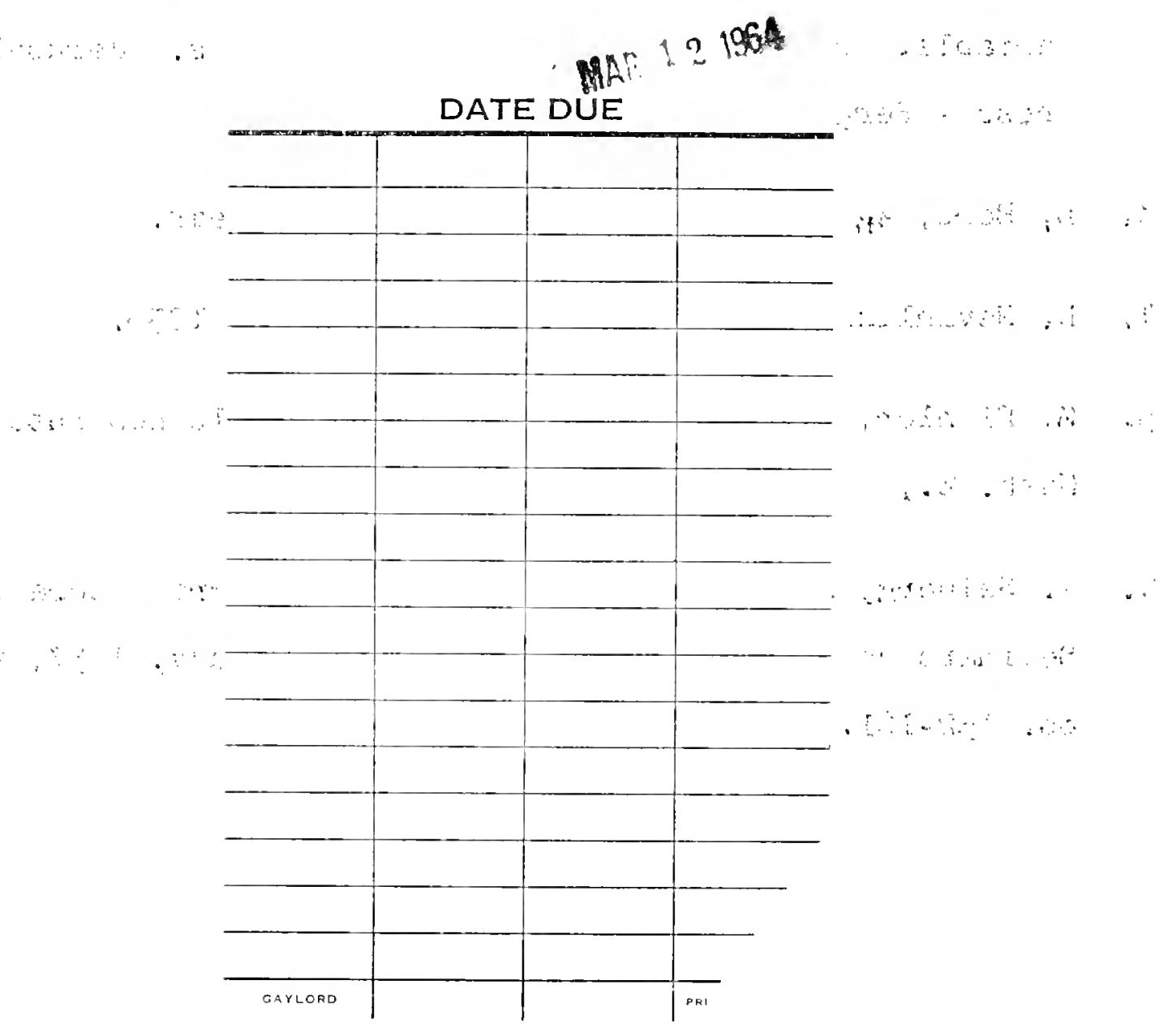




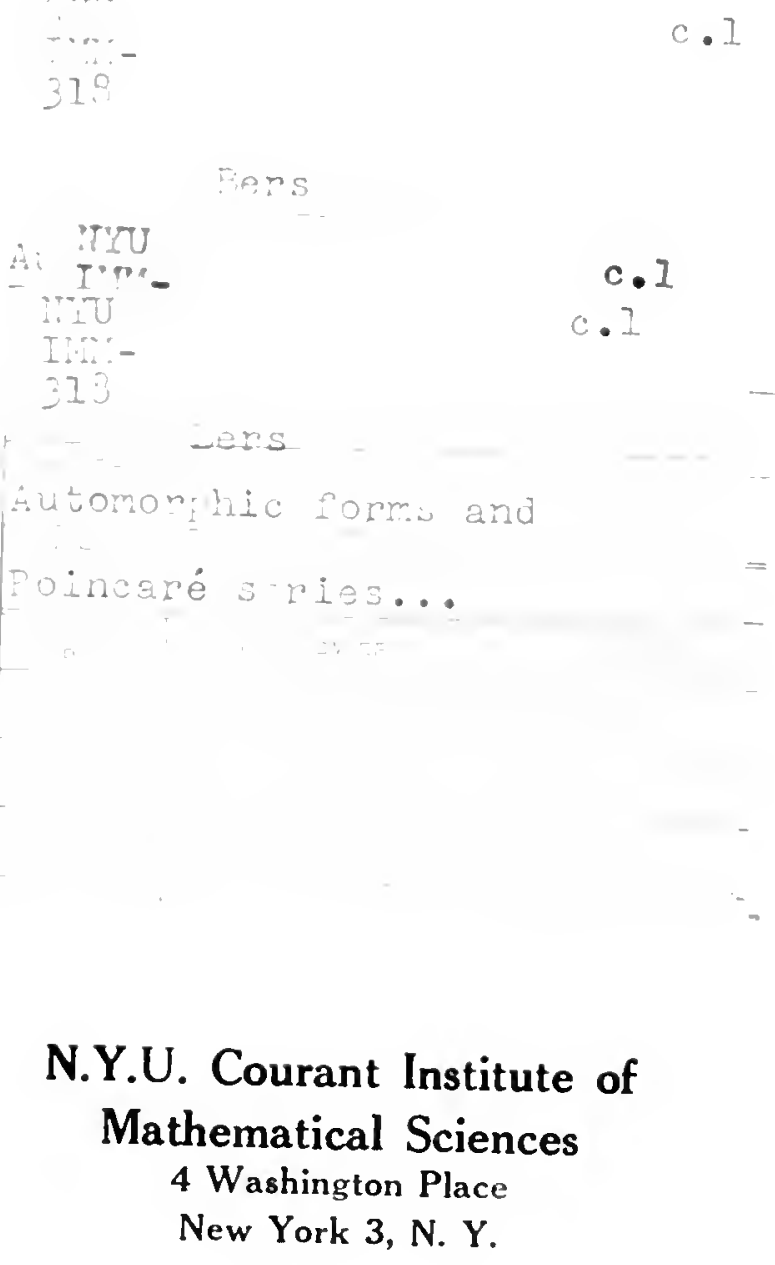




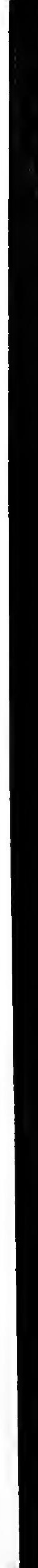

